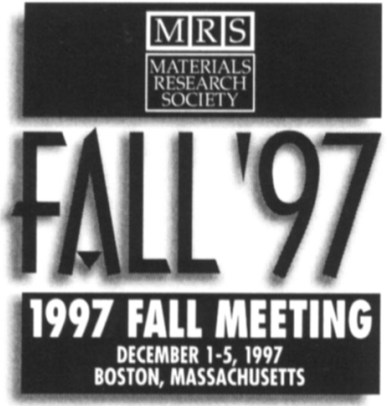

The 1997 MRS Fall Meeting demonstrates the synergy of diverse scientific communities through its 41 symposia including joint sessions and four major poster sessions. In addition to the 4,000 oral and poster presentations scheduled, an extensive exhibit, tutorials, award presentations, student activities, and other workshops round out the event. While there is a large number of technical symposia, narrowing the focus of some of them-for instance GG Functionally Graded Materials, II In Situ Process Diagnostics and Intelligent Materials Processing, and LL High-ThermalConductivity Materials: Fundamentals and Applications-gives MRS the opportunity to offer a range of new topics. The tightly enforced abstract deadline with an extra weekend given for submissions via the World Wide Web generated $64 \%$ electronic submissions, up from $19 \%$ for the Fall 1996 and Spring 1997 Meetings.

Clusters of symposia are organized by related topics. For example, representing a growing facet of MRS, soft condensed matter incorporating polymers and biomaterials will be covered in Symposia $\mathrm{J}-\mathrm{O}$. The largest component of this cluster, Symposium J, Electrical, Optical and Magnetic Properties of Organic SolidState Materials, is a massive and growing basic research area where recent results are starting to demonstrate real applications. Growing interest in this topic has been sparked by extremely encouraging early data from exploratory demonstrations. New topics in this cluster include Materials Science of the Cell (Symposium $\mathrm{K}$ ) and Polymers in Orthopedics (Symposium $\mathrm{O}$ ).

Another strength of the meeting is its focus on modeling and simulation (Symposia P-S). The materials community maintains a broad consensus for the need for modeling of complex materials and structures, synthesis and processing. This new trend focused on modeling extends from the atomistic level across length scales to cover technologically oriented topics, including a new focus on Semiconductor and Device Performance Modeling (Symposium Q).

Symposia $T$ through $W$ cover complex oxide materials including superconduc- tors, ferroelectric materials, magnetic materials, and synthesis and processing. The meeting comes at a critical time for ferroelectric thin films. Implementation of ferroelectrics into silicon technology is widely expected in the near term. In addition, the pursuit of ferroelectrics for integrated circuits has catalyzed research into a number of related applications.

In the area of semiconductors (Symposia D through I), a series of advanced semiconductor topics "off the SIA (Semiconductor Industry Association) roadmap" are highlighted. These include specialized advanced applications in power devices, detectors, and integrated optoelectronics on silicon. The area of nitrides for optoelectronics continues to show rapid progress as the materials move closer to critical large-volume applications such as data storage and as understanding of the materials grows deeper. Exemplifying the developments in this area, Symposium D on nitride semiconductors is prominent in the semiconductor cluster of this meeting. This symposium is dedicated to Jacques Pankove, inventor of the GaN LED while working at RCA Laboratories in the late 1960s. A plenary lecture by I. Akasaki will reminisce about Pankove's work.

Characterization, a core focus of MRS, covers some new approaches, for instance holography (Symposium HH) and nondestructive characterization of materials in aging systems (Symposium JJ).

Another mainstay of MRS, thin-films and more recently the area of nanostructures, is represented by symposia covering morphology, mircostructure, phase transformations, self-assembly, disordered films, irradiation of materials, and mechanical properties. Thin films and surface properties are increasingly important across a wide range of applications. In ceramic compaction, the surfaces of the ultrafine powders dominate behavior during sintering and other processes. On a fundamental level, quantum dots can be formed in arrays that can be adjusted continuously to change the electrical properties of such an "artificial crystal" from insulating to metallic. Symposia will highlight a number of unifying themes emerging in the study and understanding of the physical processes involved in these areas.

Other new and revisited topics include Electrochemical Energy Storage (Symposium Y), Catalytic Materials (Symposium $\mathrm{Z}$ ), and Advances in Materials for Cementitious Composites (Symposium MM). Symposium BB, Thermal Spray Coating, presents an opportunity to look at a particularly technologically relevant topic (applicable to aerospace, automotive, power oil and gas, and chemical communities), and try to understand the scientific issues behind it, starting first by measuring and understanding the role of parameters such as particle size, velocity, and temperature. This symposium is complemented by Symposium CC on Particulate Coatings. Symposium DD gives a critical overview of the area of materials under high pressure, which has continued to evolve since last covered at MRS.

The final symposium in the program, Symposium OO, is a two-day workshop on materials education covering innovative curricula and courses, visualization and interactive learning through use of computers, and teaching assessment. Tuesday and Wednesday morning sessions both end with panel discussions, one on needs and opportunities in materials education and the other on core curriculum to make a materials professional.

Symposium X, Frontiers of Materials Research, presents a series of authoritative reviews for the nonspecialist. Symposium $X$, presented between noon and 1:30 p.m., Tuesday through Thursday, draws from the technical content of the meeting, with an emphasis on soft condensed matter and electronic materials. Specific topics cover photoresist, sea shells, genetically engineered materials, hydrogels as biosensors, switchable mirrors from metal hydrides, and the MRS Medal award talk on III-Nitride Lasers and Optoelectronic Devices.

\section{Special Events}

The plenary session scheduled for Monday at 6:00 p.m. will feature Harry Y. McSween, Jr., professor in the Department of Geological Sciences, University of Tennessee, presenting, "Materials Research Via Mars Pathfinder." He will 
report findings from this mission based on chemical analysis with the alpha-proton-x-ray spectrometer (APXS), provided by the Max-Planck-Institut für Chemie in Germany. See the article on McSween elsewhere in this issue.

The Von Hippel Award, David Turnbull Lectureship, MRS Medal, and Graduate Student Awards will be presented at the MRS Award Ceremony Wednesday evening, December 3, at 6:00 p.m., in the Boston Marriott Hotel. After the ceremony, the Von Hippel Award recipient, Gabor A. Somorjai of the University of California-Berkeley, will give a lecture, which will be followed by a wine and cheese reception. Merton $C$. Flemings of the Massachusetts Institute of Technology will be presented with the David Turnbull Lectureship and Shuji Nakamura of Nichia Chemical Industries, Inc. will receive the MRS Medal. See full profiles of award recipients elsewhere in this issue.

The Society presents the Graduate Student Award to graduate students who are presenting significant and timely research at the 1997 Fall Meeting as an author or co-author of a symposium paper. The winners, all of whom must be presenting papers at the conference, will be chosen from Award Finalists' Special Talk Session held Monday, December 1.

The 1997 MRS Fall Meeting Chairs will recognize the best presentations at the four evening poster sessions to be held in the Marriott, Sheraton, and Westin Hotels, December 1-4. A prize of $\$ 500$ will be awarded by the Chairs to the presenting author(s) of the winning paper(s) at each evening's poster session. The Meeting Chairs will select the winners on the basis of the poster's technical content, appearance, graphic excellence, and presentation quality. Winning posters will be displayed for the remainder of the meeting. Posters were honored for the first time at the 1997 MRS Spring Meeting.

Available only to meeting registrants, symposia tutorials will concentrate on new, rapidly breaking areas of research and are designed to encourage the exchange of information by meeting attendees during the symposium. The majority of them are planned for Sunday, November 30, with one scheduled for Tuesday, December 2.

A seminar - tentatively scheduled for Tuesday 5:30-7:30 p.m. - is planned to give an overview of National Science Foundation (NSF) activities in support of materials research and education, focusing primarily on the activities of the Division of Materials Research. NSF staff will give short presentations on programs, proposal submission, and the evaluation process. A question and answer session will follow. Drop-in ses- sions are being planned later in the week for attendees to informally meet with program officers.

A major exhibit encompassing the full spectrum of equipment, instrumentation, products, software, publications, and services will be held Tuesday afternoon through Thursday in the Boston Marriott, Westin, and Sheraton Hotels, including a reception Tuesday evening 5:00-6:30 p.m.

For job seekers, a career workshop is tentatively scheduled for Tuesday, December 2, 4:30 p.m.- 8:00 p.m. and a job center tentatively set to be available 8:00 a.m.-5:00 p.m., Tuesday through Thursday, December 2-4. A student mixer will be held for graduate students and members of MRS University Chapter. Also Chapter officers and faculty advisors are invited to attend a meeting of MRS University Chapter representatives to compare notes on recent activities and brainstorm on new projects and issues of common concern.

See the following pages for a matrix of symposia sessions, profiles of exhibitors, tutorial descriptions, a registration form, and other information. For a copy of the program or to register, contact MRS at 412-779-3003; fax 412-779-8313; e-mail info@mrs.org, or see the MRS Website http:/ / www.mrs.org/ for information.

\section{Location, Lodging \& Travel}

\section{Hotels}

A block of rooms has been reserved for MRS meeting attendees at the Boston Marriott, Westin, and Sheraton Boston Hotels. Call your preferred hotel and refer to the Materials Research Society's meeting to receive the special rates, or fax the hotel.

DEADLINE FOR HOTEL RESERVATIONS: NOVEMBER 1, 1997

Boston Marriott/Copley Place

110 Huntington Avenue, Boston, MA 02116

800-228-9290; 617-236-5800 (Direct)

Fax 617-236-5885

Rate: $\$ 126 /$ Single $^{*} \bullet \$ 140 /$ Double $^{*}$

Westin Hotel/Copley Place

10 Huntington Avenue, Boston, MA 02116

800-228-3000; 617-262-9600 (Direct)

Fax 617-424-7483

Rate: $\$ 129 /$ Single ${ }^{\star} \bullet \$ 146 /$ Double $^{\star}$

Sheraton Boston Hotel and Towers

Prudential Plaza

39 Dalton Street, Boston, MA 02199

617-236-2000 (Main Desk); 617-236-2020 (Reservations)

Fax 617-236-6095

Rate: $\$ 118 /$ Single* $^{\star} \bullet \$ 129 /$ Double*

* plus Massachusetts tax

\section{Alternate Lodging}

A list of alternate lodging accommodations can be obtained via e-mail (info@mrs.org) or the MRS Website (http://www.mrs.org/).

\section{Airline Transportation \\ Due to recent changes in the airline industry, it is recommended you check with your preferred airline carrier or travel agent when making 1997 Fall Meeting travel arrangements.}

\section{Local Transportation}

Shuttle service to the Boston Marriott, Westin, and Sheraton Boston Hotels from Logan International Airport departs every halfhour, 7:00 a.m.-7:00 p.m., from the designated shuttle stop in front of each terminal.

There is a free shuttle from airport terminals to the airport subway station (The "T"). Copley Station is within one block of the Boston Marriott, Westin, and Sheraton Hotels on the "Green Line."

\section{Parking}

A parking garage is adjacent to the meeting hotels.

\section{Child Care}

Check with the Concierge Desk at the individual hotels for a comprehensive roster of licensed and bonded sitters. 


\begin{tabular}{|c|c|c|c|c|c|c|c|c|}
\hline \multirow{2}{*}{\multicolumn{2}{|c|}{ SYMPOSIUM }} & \multirow[b]{2}{*}{ LOCATION } & \multicolumn{3}{|c|}{ MONDAY, DECEMBER 1} & \multicolumn{3}{|c|}{ TUESDAY, DECEMBER 2} \\
\hline & & & a.m. & p.m. & eve. ${ }^{*}$ & a.m. & p.m. & eve.* \\
\hline A: & $\begin{array}{l}\text { Evolution of Surf. Morph. \& } \\
\text { Thin-Film Microstructure }\end{array}$ & $\begin{array}{l}\text { Essex Center } \\
\text { (W) }\end{array}$ & A1: Oxide Films \& Surfaces & A2: Heteroepitaxy & $\begin{array}{l}\text { A3: Posters } \\
(M)\end{array}$ & A4: Polycrystalline Texture & A5: Surface Dynamics & $\begin{array}{l}\text { A6: Posters } \\
\text { (M) }\end{array}$ \\
\hline B: & $\begin{array}{l}\text { Phase Transform. \& Sys. } \\
\text { Driven Far From Equilibrium }\end{array}$ & $\begin{array}{l}\text { America South } \\
\text { (W) }\end{array}$ & B1: Solidification I & $\begin{array}{l}\text { B2: Solidification II } \\
\text { B3: Nucleation }\end{array}$ & & $\begin{array}{l}\text { B4: Microstructural Evolution \& } \\
\text { Stress Effects }\end{array}$ & B5: Solid State Transformations & $\begin{array}{l}\text { B6: Posters } \\
\text { (W) }\end{array}$ \\
\hline C: & $\begin{array}{l}\text { Self-Organized } \\
\text { Nanostructures }\end{array}$ & $\begin{array}{l}\text { Essex West } \\
\text { (W) }\end{array}$ & & & & & & \\
\hline $\mathrm{D}:$ & Nitride Semiconductors & $\begin{array}{l}\text { Salon F } \\
(M)\end{array}$ & $\begin{array}{l}\text { D1: Plenary \& MOVPE I } \\
\text { D2: MOVPE ॥ }\end{array}$ & $\begin{array}{l}\text { D3: Characterization I } \\
\text { D4: Characterization II }\end{array}$ & $\begin{array}{l}\text { D5: Posters } \\
\text { (M) }\end{array}$ & $\begin{array}{l}\text { D6: Lasing \& Optical } \\
\text { Characterization } \\
\text { D7: TEM }\end{array}$ & $\begin{array}{l}\text { D8: Selective Area Epitaxy } \\
\text { D9: Processing }\end{array}$ & $\begin{array}{l}\text { D10: Posters } \\
\text { (M) }\end{array}$ \\
\hline $\mathrm{E}$ & $\begin{array}{l}\text { Power Semiconductor } \\
\text { Materials and Devices }\end{array}$ & $\begin{array}{l}\text { Wellesley } \\
\text { (M) }\end{array}$ & & $\begin{array}{l}\text { E1: Applications for Power } \\
\text { Electronics }\end{array}$ & & E2: Diamond-Based Devices & E3: Devices \& Processing & $\begin{array}{l}\text { E4: Posters } \\
(M)\end{array}$ \\
\hline $\mathrm{F}$. & $\begin{array}{l}\text { Infrared Applications of } \\
\text { Semiconductors II }\end{array}$ & $\begin{array}{l}\text { Salon J/K } \\
\text { (M) }\end{array}$ & $\begin{array}{l}\text { F1: Antimonide-Related } \\
\text { Materials-Growth, } \\
\text { Characterization, \& Analysis }\end{array}$ & F2: Antimonide-Related Devices & & F3: Innovative Infrared Devices & F4: Infrared Detectors I & $\begin{array}{c}\text { F5: Posters } \\
\text { (M) }\end{array}$ \\
\hline G: & $\begin{array}{l}\text { Thin-Film Structures for } \\
\text { Photovoltaics }\end{array}$ & $\begin{array}{c}\text { Suffolk } \\
\text { (M) }\end{array}$ & & & & G1: III-V Based Thin Films & Tutorlal Session"* & \\
\hline $\mathrm{H}:$ & $\begin{array}{l}\text { Materials and Devices for } \\
\text { Silicon-Based } \\
\text { Optoelectronics }\end{array}$ & $\begin{array}{l}\text { Simmons } \\
(\mathrm{M})\end{array}$ & $\begin{array}{l}\text { H1: Si-Based Integr. Optoelec.- } \\
\text { State of Art \& Perspectives } \\
\text { H2: Properties \& Applications of } \\
\text { Si Nanocrystals }\end{array}$ & $\begin{array}{l}\text { H3: Waveguide Materials \& } \\
\text { Devices } \\
\text { H4: Posters-Silicon } \\
\text { Nanocrystals \& Quantum Wells }\end{array}$ & & $\begin{array}{l}\text { H5: Integrated \& Discrete Infrared } \\
\text { Devices I-Light Sources } \\
\text { H6: Integr/Discrete Infrared Dev. } \\
\text { II-Detect. Amplif. \& Modulators }\end{array}$ & $\begin{array}{l}\text { H7: Devices \& Matls. Prop. for } \\
\text { Visible \& Near IR Light Emiss. } \\
\text { H8: Posters - Matls. \& Devices } \\
\text { for IR \& Visible Light Emission }\end{array}$ & \\
\hline : & $\begin{array}{l}\text { Semiconductors for Room- } \\
\text { Temperature Radiation } \\
\text { Detector Applications II } \\
\text { Sunday Tutorial Session** }\end{array}$ & $\begin{array}{l}\text { Salon } H / I \\
\text { (M) }\end{array}$ & $\begin{array}{l}\text { 11: Cadmium Zinc Telluride } \\
\text { Growth } \\
\text { 12: Cadmium Zinc Telluride } \\
\text { Characterization 1-Optical Prop. }\end{array}$ & $\begin{array}{l}\text { 13: Mercuric Iodide } \\
\text { 14: Cadmium Zinc Telluride } \\
\text { Structures 1-Imagers }\end{array}$ & & $\begin{array}{l}\text { 15: Cadmium Zinc Telluride } \\
\text { Fabrication \& Processing } \\
\text { 16: Cadmium Zinc Telluride } \\
\text { Characterization II }\end{array}$ & $\begin{array}{l}\text { 17: Device Modeling \& } \\
\text { Calculational Studies } \\
\text { 18: Cadmium Zinc Telluride } \\
\text { Structures II }\end{array}$ & $\begin{array}{l}\text { 19: Posters } \\
\text { (M) }\end{array}$ \\
\hline J: & $\begin{array}{l}\text { Electrical, Optical \& } \\
\text { Magnetic Properties of Org. } \\
\text { Solid-State Materials IV }\end{array}$ & $\begin{array}{l}\text { Salon G } \\
\text { (M) }\end{array}$ & J1: Light-Emitting Organics & $\begin{array}{l}\text { J2: Nonlinear Optical/ } \\
\text { Photorefractive Materials I }\end{array}$ & $\begin{array}{l}\text { J3: Posters } \\
\text { (M) }\end{array}$ & J4: Conjugated Polymers & J5: Supramolecular Engineering & $\begin{array}{l}\text { J6: Org. Metals } \\
\text { Magnetic Matls } \\
\text { SALON H/ (M) }\end{array}$ \\
\hline K: & Materials Science of the Cell & $\begin{array}{l}\text { Grand Ballroom } \\
\text { (S) }\end{array}$ & K1: Motility \& Force Generation & K2: Cytoskeletal Polymers & & $\begin{array}{l}\text { K3/L3: Proteins-Adsorption \& } \\
\text { Interactions with Membranes } \\
\text { REPUBLIC B (\$) }\end{array}$ & $\begin{array}{l}\text { K4a/L4a: Biomedical \& Techno- } \\
\text { logical Applications } \\
\text { REPUBLIC B (S) } \\
\text { K4: Biomed. \& Tech. Appl: }\end{array}$ & $\begin{array}{l}\text { K5: Posters } \\
\text { (S) }\end{array}$ \\
\hline L & $\begin{array}{l}\text { Complex Fluids and } \\
\text { Biomaterials }\end{array}$ & $\begin{array}{l}\text { Republic B } \\
\text { (S) }\end{array}$ & L1: Colloidal Systems & L2: Liquid Crystals & & $\begin{array}{l}\text { L3/K3: Proteins-Adsorption \& } \\
\text { Interactions with Membranes }\end{array}$ & $\begin{array}{l}\text { L4a/K4a: Biomedical \& Techno- } \\
\text { logical Applications } \\
\text { L4: Biomed. \& Tech. Appl. }\end{array}$ & $\begin{array}{l}\text { L5: Posters } \\
\text { (S) }\end{array}$ \\
\hline M: & $\begin{array}{l}\text { Adv. in Poly. Matrix Comp.- } \\
\text { Microscopic to Macroscopic }\end{array}$ & $\begin{array}{c}\text { Independence } \\
\text { Ctr (S) }\end{array}$ & $\begin{array}{l}\text { M1: Composite Synthesis \& } \\
\text { Processing }\end{array}$ & $\begin{array}{l}\text { M2: Composite Tailored } \\
\text { Interfaces }\end{array}$ & & M3: Nanocomposites & M4: Fract/Percolation in Comp. & $\begin{array}{l}\text { M5: Posters } \\
\text { (S) }\end{array}$ \\
\hline N: & $\begin{array}{l}\text { Polymers in Confined } \\
\text { Spaces }\end{array}$ & $\begin{array}{c}\begin{array}{c}\text { Constitution } \\
\text { (S) }\end{array} \\
\end{array}$ & N1: Nanocomposite Materials & $\begin{array}{l}\text { N2: Dynamics of Confined } \\
\text { Systems I }\end{array}$ & & $\begin{array}{l}\text { N3: Dynamics of Confined } \\
\text { Systems II }\end{array}$ & N4: Grafted Chains & $\begin{array}{l}\text { N5: Posters } \\
\text { (S) }\end{array}$ \\
\hline 0: & Polymers in Orthopedics & $\begin{array}{c}\text { Independence W } \\
\text { (S) }\end{array}$ & & & & & & \\
\hline P: & $\begin{array}{l}\text { Modeling Across Length } \\
\text { Scales for Materials } \\
\text { Development }\end{array}$ & $\begin{array}{l}\text { Gardner } \\
\text { (S) }\end{array}$ & $\begin{array}{l}\text { P1: Potentials for Materials } \\
\text { Development Through Modeling } \\
\text { \& Simulation }\end{array}$ & $\begin{array}{l}\text { P2: Intermetallics-Modeling \& } \\
\text { Development }\end{array}$ & & $\begin{array}{l}\text { P3: Microstructure Modeling for } \\
\text { Applications I } \\
\text { P4: Posters }\end{array}$ & $\begin{array}{l}\text { P5: Microstructure Modeling for } \\
\text { Applications II } \\
\text { P6: Posters }\end{array}$ & \\
\hline a: & $\begin{array}{l}\text { Semicond. Process \& } \\
\text { Device Perform. Modeling }\end{array}$ & $\begin{array}{c}\text { Independence E } \\
\text { (S) }\end{array}$ & & & & $\begin{array}{l}\text { Q1: Semiconductor Bulk Process } \\
\text { Modeling I }\end{array}$ & $\begin{array}{l}\text { Q2: Semiconductor Bulk } \\
\text { Process Modeling II } \\
\end{array}$ & $\begin{array}{l}\text { Q3: Posters } \\
\text { (S) }\end{array}$ \\
\hline R: & $\begin{array}{l}\text { Tight-Binding Approach to } \\
\text { Computational Mat. Science }\end{array}$ & $\begin{array}{c}\text { Fairfax A } \\
\text { (S) }\end{array}$ & $\begin{array}{l}\text { R1: First-Principles Tight } \\
\text { Binding I }\end{array}$ & $\begin{array}{l}\text { R2: First-Principles Tight } \\
\text { Binding II }\end{array}$ & & R3: Semi-Empirical Tight Binding I & $\begin{array}{l}\text { R4: Semi-Empirical Tight } \\
\text { Binding II }\end{array}$ & $\begin{array}{c}\text { R5: Posters } \\
\text { (S) }\end{array}$ \\
\hline S: & $\begin{array}{l}\text { Microscopic Simul. of Interf. } \\
\text { Phenomena in Solids \& Liq. }\end{array}$ & $\begin{array}{c}\text { Commonwealth } \\
\text { (S) }\end{array}$ & S1: Properties of Liquid Surfaces & S2: Properties of Solid Surfaces & & S3: Structure of Interfaces & $\begin{array}{l}\text { S4: Structure \& Magnetic } \\
\text { Properties of Interfaces }\end{array}$ & $\begin{array}{c}\text { S5: Posters } \\
\text { (S) }\end{array}$ \\
\hline $\mathrm{T}:$ & $\begin{array}{l}\text { Stability of High- } \mathrm{T}_{\mathrm{c}} \\
\text { Superconductors }\end{array}$ & $\begin{array}{c}\text { Salon E } \\
(\mathrm{M})\end{array}$ & T1: Thin Film Applications & T2: Microwave Properties & $\begin{array}{c}\text { T3: Posters } \\
(\mathbf{M})\end{array}$ & T4: HTS Thin Films & T5: Josephson Junctions & \\
\hline U: & $\begin{array}{l}\text { Ferroelectric Thin Films VI } \\
\text { Sunday Tutorial Session** }\end{array}$ & $\begin{array}{c}\text { Salon } A / B \\
(M)\end{array}$ & $\begin{array}{l}\text { U1: High-Permittivity Materials } \\
\text { for DRAMs }\end{array}$ & U2: Domains \& Size Effects & $\begin{array}{l}\text { U3, U4: Posters } \\
\text { (M) }\end{array}$ & U5: Barriers \& Electrodes I & U6: Barriers \& Electrodes II & $\begin{array}{l}\text { U7: Posters } \\
\text { (M) }\end{array}$ \\
\hline V: & Metallic Magnetic 0xides & $\begin{array}{l}\text { Prov/Orleans } \\
\text { (M) }\end{array}$ & $\begin{array}{l}\text { V1: Materials Processing } \\
\text { V2: Posters }\end{array}$ & V3: Invited Session & & V4: Characterization I & $\begin{array}{l}\text { V5: Characterization II } \\
\text { V6: Posters }\end{array}$ & \\
\hline W: & $\begin{array}{l}\text { Chemical Aspects of } \\
\text { Electronic Ceramics Proc. } \\
\text { Sunday Tutorial Session** }\end{array}$ & $\begin{array}{l}\text { Salon } C / D \\
\text { (M) }\end{array}$ & $\begin{array}{l}\text { W1: New Directions in Chemical } \\
\text { Processing of Electronic } \\
\text { Ceramics }\end{array}$ & $\begin{array}{l}\text { W2: MOCVD of Electronic } \\
\text { Ceramics I-0xides }\end{array}$ & & W3: Deposition & $\begin{array}{l}\text { W4: Superconductors } \\
\text { W5: MOCVD of Electronic } \\
\text { Ceramics II-Processing }\end{array}$ & $\begin{array}{l}\text { W6: Posters } \\
\text { (M) }\end{array}$ \\
\hline $\mathrm{x}:$ & $\begin{array}{l}\text { Frontiers of Materials } \\
\text { Research }\end{array}$ & $\begin{array}{c}\text { Salon E } \\
\text { (M) }\end{array}$ & & & & & $\mathrm{x}_{1}$ & \\
\hline Y: & $\begin{array}{l}\text { Matls. for Electrochemical } \\
\text { Energy Stor. \& Conversion II }\end{array}$ & $\begin{array}{l}\text { America Center } \\
\text { (W) }\end{array}$ & $\begin{array}{l}\text { Y1: Lithium Ion Rechargeable } \\
\text { Batteries-Genl \& Modeling }\end{array}$ & Y2: Fuel Cells-1 & & $\begin{array}{l}\text { Y3: Lithium Ion Rechargeable } \\
\text { Batteries - Cathode Materials I }\end{array}$ & Y4: Fuel Cells-2 & \\
\hline $\mathbf{z}$ & $\begin{array}{l}\text { Recent Advances in } \\
\text { Catalytic Materials }\end{array}$ & $\begin{array}{l}\text { Hampton } \\
\text { (S) }\end{array}$ & & & & Z1: Oxide Catalysts I & Z2: Metal Catalysts & $\begin{array}{l}\text { 23: Posters } \\
\text { (S) }\end{array}$ \\
\hline AA: & $\begin{array}{l}\text { Covalently Bonded } \\
\text { Disordered Thin-Film Matls. }\end{array}$ & $\begin{array}{l}\text { St. George } \\
B / C / D(W)\end{array}$ & & & & $\begin{array}{l}\text { AA1: Structural Models in } \\
\text { Disordered Carbon }\end{array}$ & $\begin{array}{l}\text { AA2: Characterization of } \\
\text { Amorphous Carbon }\end{array}$ & \\
\hline & $\begin{array}{l}\text { Science \& Tech. of Thermal } \\
\text { Spray Matls. Processing }\end{array}$ & $\begin{array}{l}\text { Essex N/Ctr } \\
\text { (W) }\end{array}$ & & & & BB1: Processing Science - A & BB2: Processing Science - B & \\
\hline CC: & $\begin{array}{l}\text { Particulate Coatings- } \\
\text { Synth., Charac. \& Related } \\
\text { Fundamental Phenomena }\end{array}$ & $\begin{array}{l}\text { Essex East } \\
\text { (W) }\end{array}$ & $\begin{array}{l}\text { CC1: Partic. Ctigs. - Fund. Phen. } \\
\text { CG2: Particulate Coatings - } \\
\text { Synthesis \& Processing }\end{array}$ & CC3: Nanostructured Materials & $\begin{array}{l}\text { CC4: Posters } \\
\text { (M) }\end{array}$ & $\begin{array}{l}\text { CC5: Thick Films \& Particle-Based } \\
\text { Coatings } \\
\text { CC6: Part. Adhesion \& Cleaning }\end{array}$ & & \\
\hline & $\begin{array}{l}\text { High-Pressure Materials } \\
\text { Research }\end{array}$ & $\begin{array}{l}\text { Staffordshire } \\
\text { (W) }\end{array}$ & $\begin{array}{l}\text { DD1: Earth Materials at High } \\
\text { Pressure }\end{array}$ & DD2: Dynamic Compression & $\begin{array}{l}\text { DD3: Posters } \\
\text { (W) }\end{array}$ & $\begin{array}{l}\text { DD4: New Techniques-Theory \& } \\
\text { Experiments }\end{array}$ & $\begin{array}{l}\text { DD5: High-Pressure Synthesis } \\
\text { \& Superhard Materials }\end{array}$ & \\
\hline & $\begin{array}{l}\text { Electrically Based } \\
\text { Microstructural Charac. II }\end{array}$ & $\begin{array}{c}\text { Republic A } \\
\text { (S) }\end{array}$ & $\begin{array}{l}\text { EE1: Advances in Experimental } \\
\text { Techniques }\end{array}$ & $\begin{array}{l}\text { EE2: Semiconductors \& } \\
\text { Microelectronic Applications }\end{array}$ & & $\begin{array}{l}\text { EE3: Nonlinear Conduction \& } \\
\text { Device Degradation }\end{array}$ & $\begin{array}{l}\text { EE4: Composites \& Mechanical } \\
\text { Degradation }\end{array}$ & $\begin{array}{l}\text { EE5, EE6: } \\
\text { Posters (S) }\end{array}$ \\
\hline
\end{tabular}




\begin{tabular}{|c|c|c|c|c|c|c|c|}
\hline \multicolumn{3}{|c|}{ WEDNESDAY, DECEMBER 3} & \multicolumn{3}{|c|}{ THURSDAY, DECEMBER 4} & \multicolumn{2}{|c|}{ FRIDAY, DECEMBER 5} \\
\hline a.m. & p.m. & eve. * & a.m. & p.m. & eve. ${ }^{\star}$ & \begin{tabular}{l|l} 
a.m. & \\
\end{tabular} & p.m. \\
\hline $\begin{array}{l}\text { A7 : Strain Effects on Alloy \& } \\
\text { Interface Stability }\end{array}$ & $\begin{array}{l}\text { A8: Strain Effects on Interface } \\
\text { Stability }\end{array}$ & $\begin{array}{l}\text { A9, A10, A11: } \\
\text { Posters }(W)\end{array}$ & A12: Metal Growth Atomistics & $\begin{array}{l}\text { A13: Roughening During Crystal } \\
\text { Growth \& Etching }\end{array}$ & & & \\
\hline B7: Beam-Induced Transformations & B8: Amorphous Solids & & $\begin{array}{l}\text { B9: Interfaces \& Interfacial } \\
\text { Reactions }\end{array}$ & $\begin{array}{l}\text { B10: Nanophases \& Mechanical } \\
\text { Alloying }\end{array}$ & $\begin{array}{l}\text { B11: Posters } \\
\text { (W) }\end{array}$ & B12: Thin Film Transformations & \\
\hline C1: Self-Organized Nanostructures I & C2: Self-Organized Nanostructures II & & C3: Self-Organized Nanostruc. III & C4: Self-Organized Nanostruc. IV & $\begin{array}{l}\text { C5: Posters } \\
(W)\end{array}$ & & \\
\hline $\begin{array}{l}\text { D11: Bulk Crystal Growth } \\
\text { D12: Phase Separation in InGaN }\end{array}$ & $\begin{array}{l}\text { D13: MBE } \\
\text { D14: Theory }\end{array}$ & $\begin{array}{l}\text { D15: Posters } \\
\text { (M) }\end{array}$ & $\begin{array}{l}\text { D16: HVPE } \\
\text { D17: P-Type Doping \& LEDs }\end{array}$ & $\begin{array}{l}\text { D18: Characterization III } \\
\text { D19: QWs \& Heterojunctions }\end{array}$ & & $\begin{array}{l}\text { D20: Properties of GaN Lasers } \\
\text { D21: Industrial Applications of GaN } \\
\text { Lasers }\end{array}$ & \\
\hline E5: Silicon Carbide Technology & E6: Dielectrics, Processing & & E7: IGBTs and FETs & E8: Devices \& Processing Issues & & & \\
\hline $\begin{array}{l}\text { F6/l10: Materials Growth \& Doping } \\
\text { of II-VI }\end{array}$ & $\begin{array}{l}\text { F7/11: Infrared Detectors II } \\
\text { F8/112: Infrared Detectors II (Cont'd) }\end{array}$ & & $\begin{array}{l}\text { F9: Interciffusion in Quantum Well } \\
\text { Materials \& IR Applications }\end{array}$ & $\begin{array}{l}\text { F10: Nonlinear Optical and OPO } \\
\text { Materials }\end{array}$ & & & \\
\hline G2: Si-Based Thin Films - A & G3: Si-Based Thin Films - B & & G4: II-VI Gased Thin Films - A & G5: II-VI Based Thin Films - B & & G6: Thin Films - General & \\
\hline $\begin{array}{l}\text { H9: Quantum Wells \& New } \\
\text { Materials } \\
\text { H10: Photonics Devices in \& On Si }\end{array}$ & $\begin{array}{l}\text { H11: New Matl. \& Device Concepts } \\
\text { H12: Posters-Por. Si-Based Mat. } \\
\text { H13: Posters-New Matls. for } \\
\text { Integ. \& Interconn. }\end{array}$ & & & & & & \\
\hline $\begin{array}{l}\text { I10/F6: Materials Growth \& Doping } \\
\text { of II-VI } \\
\text { SALON J/K (M) }\end{array}$ & $\begin{array}{l}\text { I11/F7: Infrared Detectors II } \\
\text { SALON J/K (M) } \\
\text { I12/FB: Infrared Detectors II (Contd) } \\
\text { SALON J/K (M) }\end{array}$ & & $\begin{array}{l}\text { 113: Cadmium Telluride } \\
\text { I14: Vapor Deposited } \\
\text { Semiconductors for Detectors }\end{array}$ & $\begin{array}{l}\text { 115: Lead lodide } \\
\text { 116: Other Semiconductor } \\
\text { Detector Materials }\end{array}$ & & $\begin{array}{l}\text { I17: Group IV Semiconductors for } \\
\text { Detectors } \\
\text { 118: Radiation Damage }\end{array}$ & \\
\hline $\begin{array}{l}\text { J7: Nonlinear OpticaV Photorefrac- } \\
\text { tive Materials II }\end{array}$ & J8: Light-Emitting Polymers & $\begin{array}{l}\text { J9: Posters } \\
\text { (W) }\end{array}$ & $\begin{array}{l}\text { J10: Molecular Engineering/Liquid } \\
\text { Crystal Display }\end{array}$ & J11: Conjugated Polymers & & $\begin{array}{l}\text { J12: Nonlinear Optical/ } \\
\text { Photorefractive Materials III }\end{array}$ & $\begin{array}{l}\text { J13: Light- } \\
\text { Emitting Matls }\end{array}$ \\
\hline K6: Membranes \& Vesicles & $\begin{array}{l}\text { K7: Supported Bilayers } \\
\text { K8: Cells }\end{array}$ & & $\begin{array}{l}\text { K9: Cell Adhesion, Motility, } \\
\text { Transport }\end{array}$ & $\begin{array}{l}\text { K10: Single Molecular Mechanics } \\
\text { \& DNA Manipulations }\end{array}$ & & & \\
\hline L6: Dynamics in Complex Fluids I & L7: Dynamics in Complex Fluids II & & $\begin{array}{l}\text { L8: Polymer \& DNA Complexes with } \\
\text { Oppositely Charged Amphiphiles }\end{array}$ & $\begin{array}{l}\text { L9: Microemulsions \& Theory of } \\
\text { Amphiphile Phases }\end{array}$ & & L10: Neutral \& Charged Polymers & $\begin{array}{l}\text { L11: Mixed \& } \\
\text { Poly. Amph. }\end{array}$ \\
\hline $\begin{array}{l}\text { M6: Composite Fracture \& } \\
\text { Durability }\end{array}$ & M7: Composite Interface Adhesion & & $\begin{array}{l}\text { M8: Toughness, Characterization, } \\
\text { Sensing }\end{array}$ & $\begin{array}{l}\text { M9: Composite Properties vs } \\
\text { Structure }\end{array}$ & & & \\
\hline N6: Adhesion & N7: Polyelectrolytes \& Biopolymers & & $\begin{array}{l}\text { N8: Phase Transitions in Confined } \\
\text { Geometries I }\end{array}$ & $\begin{array}{l}\text { N9: Phase Transitions in Confined } \\
\text { Geometries II }\end{array}$ & & N10: Adsorption & $\begin{array}{l}\text { N11: } \\
\text { Session } 11 \\
\end{array}$ \\
\hline 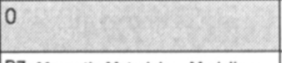 & $\begin{array}{l}\text { 01: Tissue Engineering } \\
\text { 02: Resorbables \& Implants }\end{array}$ & & $\begin{array}{l}\text { 03: Bone Grafts \& Bone Cement } \\
\text { 04: Drug Delivery }\end{array}$ & \begin{tabular}{l|l} 
05: Polyethylene \\
06: Posters
\end{tabular} & & & \\
\hline $\begin{array}{l}\text { P7: Magnetic Materials-Modeling } \\
\text { \& Development }\end{array}$ & $\begin{array}{l}\text { P8: Semiconducting Materials- } \\
\text { Modeling \& Development }\end{array}$ & & $\begin{array}{l}\text { Pg: Modeling Dislocations \& } \\
\text { Dislocation Dynamics }\end{array}$ & & & & \\
\hline $\begin{array}{l}\text { Q4: Semiconductor Equipment } \\
\text { Modeling }\end{array}$ & $\begin{array}{l}\text { Q5: Semicond. Topog. Modeling } \\
\text { Q6: Semicond. Charac. \& Device } \\
\text { Modeling }\end{array}$ & & & & & & \\
\hline R6: Tight-Binding Simulations I & R7: Tight-Binding Simulations II & & & & & & \\
\hline $\begin{array}{l}\text { S6: Mechanical Properties of } \\
\text { Interfaces }\end{array}$ & $\begin{array}{l}\text { S7: Transport Properties \& Phase } \\
\text { Transitions }\end{array}$ & & S8: Thin Films \& Nanostructures & $\begin{array}{l}\text { S9: Chemical Properties of } \\
\text { Interfaces }\end{array}$ & & & \\
\hline $\begin{array}{l}\text { T6: Superconductivity in Japan, } \\
\text { U.S., \& Europe }\end{array}$ & $\begin{array}{l}\text { T7: Conductor Applications \& } \\
\text { Materials }\end{array}$ & $\begin{array}{l}\text { T8: Posters } \\
\text { (M) }\end{array}$ & T9: Melt-Textured Materials & T10: Flux Pinning \& AC Loss & \begin{tabular}{c|c} 
T11: Posters \\
(M)
\end{tabular} & T12: Bi Conductors & $\begin{array}{l}\text { T13: Y \& } \\
\text { Other Conduct }\end{array}$ \\
\hline U8: Bi-Layered Ferroelectrics & U9: Pb-Based Ferroelectrics & $\begin{array}{l}\text { U10, U11: } \\
\text { Posters (M) }\end{array}$ & U12: Microwave \& Optical Devices & $\begin{array}{l}\text { U13: Materials for Piezoelectric } \\
\text { MEMS }\end{array}$ & $\begin{array}{l}\text { U14, U15, U16 } \\
\text { Posters (M) }\end{array}$ & $\begin{array}{l}\text { U17: Ferroelectric Thin-Film } \\
\text { Devices }\end{array}$ & \\
\hline V7: Theory \& Devices & V8: Devices \& Multilayers & & V9: Physical Properties I & $\begin{array}{l}\text { V10: Physical Properties II } \\
\text { V11: Posters }\end{array}$ & & & \\
\hline $\begin{array}{l}\text { W7: MOCVD of Electronic Ceramics } \\
\text { III-Precursors } \\
\text { W8: Silicon Dielectrics }\end{array}$ & $\begin{array}{l}\text { W9: Novel Processing of Electronic } \\
\text { Ceramics }\end{array}$ & & $\begin{array}{l}\text { W10: Processing of Silicon Carbide } \\
\text { \& Diamond }\end{array}$ & $\begin{array}{l}\text { W11/118: Intelligent Processing of } \\
\text { Electronic Ceramics }\end{array}$ & $\begin{array}{l}\text { W12: Panel } \\
\text { Disc--Future } \\
\text { of Electronic } \\
\text { Ceram. Proc. } \\
\end{array}$ & & \\
\hline$x$ & $x_{2}$ & & & X3 & & & \\
\hline Y5: Supercapacitors & $\begin{array}{l}\text { Y6: Matls. for Other Battery Sys. } \\
\text { Y7: Lithium Ion Rechargeable } \\
\text { Batteries - Cathode Matls. II }\end{array}$ & $\begin{array}{l}\text { Y8: Posters } \\
\text { (W) }\end{array}$ & $\begin{array}{l}\text { Y9: Battery Materials } \\
\text { Characterization }\end{array}$ & $\begin{array}{l}\text { Y10: Lithium lon Rechargeable } \\
\text { Battery-Anode Materials }\end{array}$ & & $\begin{array}{l}\text { Y11: Battery Electrolytes, } \\
\text { Interfaces, \& Passive Films }\end{array}$ & \\
\hline $\begin{array}{l}\text { Z4: Oxide Catalysts II } \\
\text { Z5: Carton-Based Catalysts }\end{array}$ & $\begin{array}{l}\text { Z6: Pillared, Layered \& Porous } \\
\text { Catalysts }\end{array}$ & & $\begin{array}{l}\text { Z7: Sulfides \& Carbides } \\
\text { Z8: Zeolites \& Related Materials }\end{array}$ & \begin{tabular}{l|l|} 
29: Acids \& Bases \\
Z10: Surface Modifications
\end{tabular} & & & \\
\hline $\begin{array}{l}\text { AA3: Amorphous Carbon Growth \& } \\
\text { Tribology }\end{array}$ & $\begin{array}{l}\text { AA4: Boron \& Carbon Nitrides I } \\
\text { AA5: Posters-Bor.\& Carb. Nit. II }\end{array}$ & & $\begin{array}{l}\text { AA6: Electron Emission I } \\
\text { AA7: Posters-Electron Emiss. II }\end{array}$ & $\begin{array}{l}\text { AA8: Electron Emission III } \\
\text { AA9: Posters-Covalently Bonded } \\
\text { Materials }\end{array}$ & & & \\
\hline $\begin{array}{l}\text { BB3: Emerging Technologies \& } \\
\text { Applications - A }\end{array}$ & $\begin{array}{l}\text { BB4: Emerging Tech. \& Applic. - B } \\
\text { B85: Science \& Technology of } \\
\text { Water-Stabilized Plasmas }\end{array}$ & & $\begin{array}{l}\text { BB6: Near-Net Fabrication } \\
\text { BB7: Mechanical Properties - A }\end{array}$ & $\begin{array}{l}\text { BB8: Mechanical Properties - B } \\
\text { BB9: Microstructures \& } \\
\text { Imperfections }\end{array}$ & & & \\
\hline \multicolumn{8}{|c|}{ (1) } \\
\hline $\begin{array}{l}\text { DD6: Hydrogen at High } \\
\text { Temperature }\end{array}$ & $\begin{array}{l}\text { DD7: Semicond. \& Supercond. at } \\
\text { High Pressures }\end{array}$ & & $\begin{array}{l}\text { DD8: Dense Solids_-Molecular \& } \\
\text { Metallic }\end{array}$ & $\begin{array}{l}\text { DD9: Metastability, Amorphization } \\
\text { \& Glasses }\end{array}$ & & & \\
\hline EE7: Dielectrics \& Ferroelectrics & $\begin{array}{l}\text { EE8: Ionic \& Miked Conducting } \\
\text { Materials }\end{array}$ & & & & & & \\
\hline
\end{tabular}




\begin{tabular}{|c|c|c|c|c|c|c|c|c|}
\hline \multirow{2}{*}{\multicolumn{2}{|c|}{ SYMPOSIUM }} & \multirow[b]{2}{*}{ LOCATION } & \multicolumn{3}{|c|}{ MONDAY, DECEMBER 1} & \multicolumn{3}{|c|}{ TUESDAY, DECEMBER 2} \\
\hline & & & a.m. & p.m. & eve.* & a.m. & p.m. & eve.* \\
\hline FF: & $\begin{array}{l}\text { Surface-Controlled } \\
\text { Nanoscale Materials for } \\
\text { High-Added-Value Applic. } \\
\text { Sunday Tutorial Session** }\end{array}$ & $\begin{array}{l}\text { America North } \\
\text { (W) }\end{array}$ & FF1: Electronic Nanomaterials & $\begin{array}{l}\text { FF2: Electronic \& Optical } \\
\text { Nanomaterials }\end{array}$ & & $\begin{array}{l}\text { FF3: Magnetic Nanomaterials \& } \\
\text { Imaging }\end{array}$ & $\begin{array}{l}\text { FF4: Nanocomposites \& } \\
\text { Coating }\end{array}$ & $\begin{array}{l}\text { FF5: Posters } \\
\text { (W) }\end{array}$ \\
\hline GG: & $\begin{array}{l}\text { Functionally Graded } \\
\text { Materials }\end{array}$ & $\begin{array}{l}\text { Essex East } \\
(\mathrm{W})\end{array}$ & & . & & $\begin{array}{l}\text { GG1: Modeling } \\
\text { ADAMS (W) }\end{array}$ & GG2: Processing I & \\
\hline HH: & $\begin{array}{l}\text { Materials Applications of } \\
\text { Electron Holography and } \\
\text { Related Techniques }\end{array}$ & $\begin{array}{l}\text { Berkeley } \\
\text { (S) }\end{array}$ & & & & & & \\
\hline it: & $\begin{array}{l}\text { In Situ Process Diag. \& } \\
\text { Intelligent Matls. Processing }\end{array}$ & $\begin{array}{l}\text { Cape Cod/ } \\
\text { Hyannis } \\
\text { (M) }\end{array}$ & & & & $\begin{array}{l}\text { II1: Sensor Technologies for } \\
\text { In-Situ Diagnostics }\end{array}$ & $\begin{array}{l}\text { I12: Semiconductor Diagnostics } \\
\text { II3: Posters-Semiconductor } \\
\text { Diagnostics }\end{array}$ & \\
\hline JJ: & $\begin{array}{l}\text { Nondestructive Charac. of } \\
\text { Materials in Aging Systems } \\
\text { Sunday Tutorial Session ** }\end{array}$ & $\begin{array}{c}\text { Fairfax B } \\
\text { (S) }\end{array}$ & $\begin{array}{l}\text { JJ1: Nondestructive } \\
\text { Characterization of Materials in } \\
\text { Aging Aerospace Structures }\end{array}$ & $\begin{array}{l}\text { JJ2: Nondestructive } \\
\text { Characterization of Aging } \\
\text { Composite \& Bonded Structures }\end{array}$ & & $\begin{array}{l}\text { JJ3/MM4: Nondestructive } \\
\text { Characterization of Cement } \\
\text { INDEPENDENCE WEST (S) }\end{array}$ & $\begin{array}{l}\text { JJ4: Characterization of } \\
\text { Radiation Damaged Materials }\end{array}$ & \\
\hline KK: & $\begin{array}{l}\text { Atomistic Mechanisms in } \\
\text { Beam Synthesis and } \\
\text { Irradiation of Materials }\end{array}$ & $\begin{array}{l}\text { Essex West } \\
\text { (W) }\end{array}$ & KK1: Defects \& Modeling & $\begin{array}{l}\text { KK2: Material Flow, Ceramic } \\
\text { Materials, \& Cluster-Beam } \\
\text { Deposition }\end{array}$ & $\begin{array}{l}\text { KK3: Posters } \\
\text { (M) }\end{array}$ & $\begin{array}{l}\text { KK4: Energetic Particle Synthesis, } \\
\text { Modeling \& Mechanical Properties }\end{array}$ & $\begin{array}{l}\text { KK5: Optical Materials, Nano- } \\
\text { clusters, Ceramics, \& Polymers }\end{array}$ & $\begin{array}{l}\text { KK6: Posters } \\
\text { (M) }\end{array}$ \\
\hline ㄴ: & $\begin{array}{l}\text { High-Thermal-Conductivity } \\
\text { Materials-Fund. \& Applic. }\end{array}$ & $\begin{array}{l}\text { Boston College } \\
\text { (M) }\end{array}$ & $\begin{array}{l}\text { LL1: Thermal Conductivity } \\
\text { Mechanisms \& Measurement }\end{array}$ & LL2: Processing \& Metallization & & $\begin{array}{l}\text { LL3: Composites-Processing \& } \\
\text { Properties }\end{array}$ & & \\
\hline MM: & $\begin{array}{l}\text { : Advances in Materials for } \\
\text { Cementitious Composites }\end{array}$ & $\begin{array}{c}\text { Independence W } \\
\text { (S) }\end{array}$ & $\begin{array}{l}\text { MM1: Waste Treatment \& } \\
\text { Utilization }\end{array}$ & $\begin{array}{l}\text { MM2: Comp/Reinforc. Concrete } \\
\text { MM3: Posters }\end{array}$ & & $\begin{array}{l}\text { MM4/JJ3: Nondestructive } \\
\text { Characterization of Cement }\end{array}$ & $\begin{array}{l}\text { MM5: Processing \& Hydration } \\
\text { MM6: Novel Systems \& Applic. }\end{array}$ & \\
\hline NN: & $\begin{array}{l}\text { Thin Films-Stresses and } \\
\text { Mechanical Properties VII }\end{array}$ & $\begin{array}{l}\text { Essex South } \\
\text { (W) }\end{array}$ & NN1: Novel Testing Methods & NN2: Low-Load Indentation & & $\begin{array}{l}\text { NN3: Modeling \& Simulations I- } \\
\text { Low-Load Indentation } \\
\text { NN4: Metallization \& Reliability }\end{array}$ & $\begin{array}{l}\text { NN5: Structural \& Mechanical } \\
\text { Stability }\end{array}$ & $\begin{array}{l}\text { NN6: Posters } \\
\text { (M) }\end{array}$ \\
\hline 00: & $\begin{array}{l}\text { Workshop on Materials } \\
\text { Education }\end{array}$ & $\begin{array}{c}\text { Regis/ } \\
\text { Boston Univ } \\
\text { (M) }\end{array}$ & & & & $\begin{array}{l}\text { 001: Innovations in Materials } \\
\text { Curricula/Assessment }\end{array}$ & $\begin{array}{l}\text { 002: Computers in Materials } \\
\text { Education }\end{array}$ & $\begin{array}{l}\text { 003: Demos- } \\
\text { Software \& } \\
\text { Teaching } \\
\text { Resources } \\
\end{array}$ \\
\hline
\end{tabular}

- Check Poster Session Locator.

-. Check Tutorial Program Matrix.

-.. Shaded Blocks: No Session

\begin{tabular}{|c|c|c|c|}
\hline \multicolumn{4}{|c|}{ MRS 1997 FALL MEETING - EVENING POSTER SESSION LOCATOR } \\
\hline Monday, 12/1 & Tuesday, $12 / 2$ & Wednesday, $12 / 3$ & Thursday, $12 / 4$ \\
\hline \multicolumn{4}{|c|}{ Boston Marriott } \\
\hline \begin{tabular}{ll} 
& \multicolumn{1}{c}{ Salons G-K } \\
D5: & Crystal Growth \\
T3: & HTS Films and Devices \\
U3: & BST/DRAM \\
U4: & Domains and Size Effects
\end{tabular} & \begin{tabular}{ll} 
& \multicolumn{1}{c}{ Salons E-G } \\
D10: & Characterization \\
E4: Power Devices and Materials \\
F5: Infrared Materials and \\
I9: Devices \\
U7: \\
W6ster Session \\
W6: Solutiodes and Barriers \\
& of Electronic Ceramics
\end{tabular} & \begin{tabular}{ll} 
& \multicolumn{1}{c}{ Salons G-K } \\
D15: & Theory, Processing and \\
& Devices \\
T8: & YBCO and Other Materials \\
U10: & Bi-Layered Ferroelectrics \\
U11: & Pb-Based Ferroelectrics
\end{tabular} & \begin{tabular}{ll} 
& \multicolumn{1}{c}{ Salon G } \\
T11: & $\begin{array}{l}\text { Bi Conductors and } \\
\text { Processing }\end{array}$ \\
U14: & Microwave/Optical Devices \\
U15: & MEMS \\
U16: & Novel Ferroelectric Devices
\end{tabular} \\
\hline
\end{tabular}

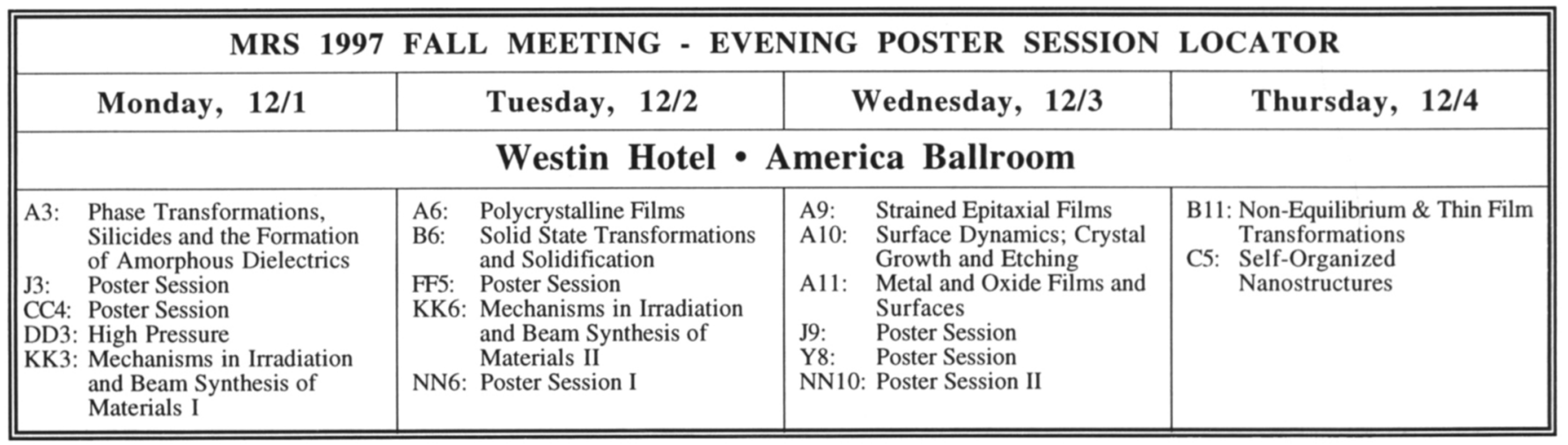

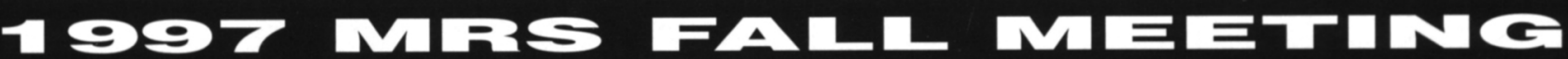 Preregistration Deadline: November 14, 1997 $412-779-3003$}




\begin{tabular}{|c|c|c|c|c|c|c|c|}
\hline \multicolumn{3}{|c|}{ WEDNESDAY, DECEMBER 3} & \multicolumn{3}{|c|}{ THURSDAY, DECEMBER 4} & \multicolumn{2}{|c|}{ FRIDAY, DECEMBER 5} \\
\hline a.m. & p.m. & eve.* & a.m. & p.m. & eve.* & a.m. & p.m. \\
\hline $\begin{array}{l}\text { FF6: Functionalization \& } \\
\text { Biomaterials }\end{array}$ & $\begin{array}{l}\text { FF7: Sintering \& Future Trends in } \\
\text { Nanostructured Materials }\end{array}$ & & & & & & \\
\hline $\begin{array}{l}\text { GG3: Processing II } \\
\text { GG4: Fracture \& Deformation I }\end{array}$ & $\begin{array}{l}\text { GG5: Fracture and Deforma- } \\
\text { tion II }\end{array}$ & & GG6: Design for Performance & $\begin{array}{l}\text { GG7: Thermal \& Electrical } \\
\text { Properties }\end{array}$ & & & \\
\hline $\begin{array}{l}\text { HH1: Electron Holography } \\
\text { Techniques }\end{array}$ & $\begin{array}{l}\text { HH2: Applications I-Electric \& } \\
\text { Magnetic Fields }\end{array}$ & & $\begin{array}{l}\text { HH3: Applications II-Materials } \\
\text { Characterization }\end{array}$ & $\begin{array}{l}\text { HH4: Related Techniques-X-Ray } \\
\text { \& Photoelectron Holography } \\
\text { HH5: Posters } \\
\end{array}$ & & & \\
\hline $\begin{array}{l}\text { 114: Sensors for Intelligent } \\
\text { Processing }\end{array}$ & $\begin{array}{l}\text { II5: Thin-Film Processes } \\
\text { II6: Posters-Thin-Film Processes }\end{array}$ & & $\begin{array}{l}\text { I17: In-Situ Diagnostics of Oxide Film } \\
\text { Processes }\end{array}$ & $\begin{array}{l}\text { 118/W11: Intelligent Processing of } \\
\text { Electronic Ceramics } \\
\text { SALON C/D (M) } \\
\end{array}$ & & $\begin{array}{l}\text { I19: In Situ Diag. of Oxide Film } \\
\text { Growth }\end{array}$ & \\
\hline $\begin{array}{l}\text { JJ5: Nondestructive Charac. of } \\
\text { Infrastructure Materials }\end{array}$ & $\begin{array}{l}\text { JJ6: New Aging Materials } \\
\text { Characterization Techniques I }\end{array}$ & & $\begin{array}{l}\text { JJ7: New Aging Materials } \\
\text { Characterization Techniques II }\end{array}$ & & & & \\
\hline \multicolumn{8}{|l|}{ KK } \\
\hline \multicolumn{8}{|l|}{ LL } \\
\hline \multicolumn{8}{|l|}{ MM7: Characterization } \\
\hline NN7: Tribological Properties & $\begin{array}{l}\text { NN8: Thin Film Adhesion } \\
\text { NN9: Deformation \& Fracture } \\
\text { Mechanisms }\end{array}$ & $\begin{array}{l}\text { NN10: Posters } \\
\text { (M) }\end{array}$ & $\begin{array}{l}\text { NN11: Stresses in Thin Films I- } \\
\text { Generation Mechanisms } \\
\text { NN12: Stresses in Thin Films II- } \\
\text { Measurement Techniques }\end{array}$ & $\begin{array}{l}\text { NN13: Modeling \& Simulations II } \\
\text { NN14: Artificially Multilayered \& } \\
\text { Superlattice Thin Films }\end{array}$ & & $\begin{array}{l}\text { NN15: Processing-Microstructure- } \\
\text { Mechanical Property Relationships }\end{array}$ & \\
\hline $\begin{array}{l}\text { 004: Innovative Courses \& } \\
\text { Programs I }\end{array}$ & $\begin{array}{l}\text { 005: Innovative Courses \& } \\
\text { Programs II/Assessment }\end{array}$ & & & & & & \\
\hline
\end{tabular}

\begin{tabular}{|c|c|}
\hline & $\begin{array}{c}\text { MRS } 1997 \text { FALL MEETING } \\
\text { EVENING POSTER SESSION LOCATOR }\end{array}$ \\
\hline \multicolumn{2}{|r|}{ Tuesday, $12 / 2$} \\
\hline & Sheraton Boston • Grand Ballroom \\
\hline $\begin{array}{l}\text { K5: } \\
\text { L5: } \\
\text { M5: } \\
\text { N5: } \\
\text { Q3: } \\
\text { R5: } \\
\text { S5: } \\
\text { Z3: } \\
\text { EE5: } \\
\text { EE6: }\end{array}$ & $\begin{array}{l}\text { Poster Session } \\
\text { Poster Session } \\
\text { Poster Session } \\
\text { Poster Session } \\
\text { Poster Session } \\
\text { Semi-Empirical Tight-Binding--Part III } \\
\text { Interfaces in Solids and Liquids } \\
\text { Catalytic Materials } \\
\text { Microelectronic Applications } \\
\text { Corrosion of Metals and Polymer/Metal Interface }\end{array}$ \\
\hline
\end{tabular}

\section{MRS FALL MEETING} Download the Hotel Reservation form from the web: http://www.mrs.org/meetings/fall97/ program_book/location.html

Deadline: November 1,1997

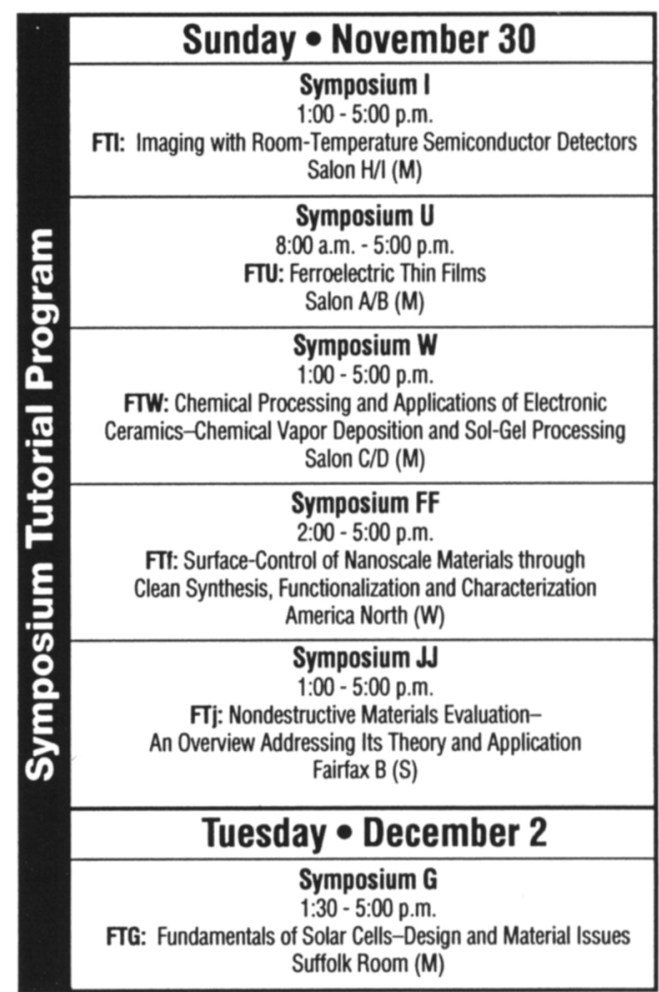

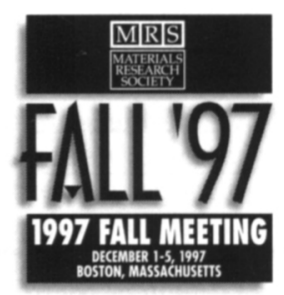

\section{For your convenience, register for the 1997 MRS Fall Meeting on the Web:} http://www.mrs.org/meetings/fall97/program_book/register.html 
W Take advantage of the speed and convenience of on-line preregistration via the MRS : Website: http://www.mrs.org/
F Fax this form to MRS Member Services, 412-779-8313, in service 24 hours every day.

Fax registration requires credit card payment.
Call MRS Member Services, 412-779-3003 between 8:00 a.m. and 5:00 p.m. EST. Telephone registration requires credit card payment; have your credit card and this form in front of you for easy reference. Return this form with payment to:

MRS Member Services 506 Keystone Drive Warrendale, PA 15086-7573 USA

\section{PREREGISTRATION DEADLINE: NOVEMBER 14, 1997}

NOTE: Please enter MRS ID No. (0..)

from your mailing label, if available.

If not known, leave box blank.

Enter MAS ID No. in box

$\begin{array}{llll}\text { Institution Type: } & \square \text { Industry } & \square \text { University } & \square \text { Government } \\ \text { This address is: } & \square \text { Business } & \square \text { Home } & \square \text { New Address }\end{array}$

\begin{tabular}{lll}
\hline Name: First & Middle
\end{tabular}

Job Title Institution

Dept/Mail Stop

P.O. Box

Street Address

\section{Postal Code 1}

City

Postal Code 2

State/Province

Zip

Country

\begin{tabular}{llll}
\hline Phone: & Country & Area/City & Local Number \\
& & & \\
\hline Fax: & Country & Area/City & Local Number
\end{tabular}

E-mail address

WwW address

MRS selectively permits use of its membership list by advertisers of products which the Society deems to be of high interest to MRS members. Please check if you do not wish to receive these mailings.

\section{A Meeting Preregistration}

\section{PAYMENT MUST ACCOMPANY PREREGISTRATION.}

Please check category and enter amount in payment section below.
$\square \quad \$ 275$ MRS Member
- \$ 75 MRS Student Member
\$105 Retired
$\square$ \$325 Nonmember
$\square \$ 105$ Unemployed
After November 14, 1997:
\\$315 MAS Member
$\square \$ 85$ Student Nonmember
$\$ 105$ Retired
$\square$ \$365 Nonmember
$\square \$ 85$ MRS Student Member
$\square 105$ Retired
$\square 105$ Unemployed

Student registration will not be processed without proof of full-time student status.

All registrations include complimentary MRS membership through December 31, 1998.

- Enter total here and in PAYMENT OPTIONS box. TOTAL \$

Symposium interest (please check all that apply):
$\square \mathrm{A} \quad \square \mathrm{B}$
$\square \mathrm{C}$
$\square \mathrm{D}$
$\square E \square F$
$\square \mathrm{G} \quad \square \mathrm{H}$
$\square \mathrm{I} \square \mathrm{J}$
$\square \mathrm{K} \quad \square \mathrm{L} \quad \square \mathrm{M}$
$\square N \square O \square P$
$\square Q$
ar $\square \mathrm{S}$
$\square T$
$\square U$
$\square \mathrm{V} \square \mathrm{W} \square \mathrm{Y}$
$\square \mathrm{Z} \square \mathrm{AA}$
$\square \mathrm{BB}$
$\square \mathrm{CC} \square \mathrm{DD} \square \mathrm{EE}$
$\square \mathrm{FF}$
$\square J J \square K K$

\section{Journal of Materials Research 1998}

Monthly Subscription at U.S. Member Rate (one per registrant) $\square \$ 80^{*}$ Monthly Subscription at Non-U.S. Member Rate (one per registrant) $\square \$ 95^{*}$ Optional Air Freight (non-U.S.) $\square \$ 76$

*Includes CD ROM version at no extra charge. Delivery early 1999.

- Enter total here and in PAYMENT OPTIONS box.

TOTAL :

\section{Proceedings (published after this meeting)}

These rates apply only to meeting attendees and MRS members. Nonmembers must contact MRS Headquarters for prices. (Cost of proceedings will increase after the meeting.)

TOTAL

NO. COPIES

B: Phase Transform. \& Systems Driven Far From Equilibrium . . . . $\$ 56 \times \ldots \ldots$ D: Nitride Semiconductors $\ldots \ldots \ldots \ldots \ldots \ldots \ldots \ldots \ldots \ldots . \$ 56 \times \ldots \ldots \ldots \ldots$

E: Power Semiconductor Materials and Devices . . . . . . . . . $\$ 50 \times \ldots \ldots$

F: Infrared Applications of Semiconductors II ............\$52 $\times \ldots \ldots \ldots$

G: Thin-Film Structures for Photovoltaics ............\$50 $\times$

$\mathrm{H}$ : Materials \& Devices for Silicon-Based Optoelectronics ......\$50 $\times$ _ $=\$$

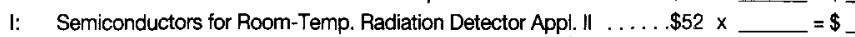

$\mathrm{J}$ : Electrical, Opt. \& Magnetic Prop. of Org. Solid-State Matls. IV .....\$56 $\times-$

$\mathrm{K}:$ Materials Science of the Cell .................. $\$ 55 \times \ldots$

$\mathrm{Q}:$ Semiconductor Process \& Device Performance Modeling $\ldots \ldots . \$ 48 \times \square=\$$

R: Tight-Binding Approach to Computational Matls. Science ....\$50 $\times$ _ $=\$$

S: Microscopic Sim. of Interfacial Phenomena in Solids \& Liq. ..... $\$ 52 \times \square$

$\mathrm{U}$ : Ferroelectric Thin Films $\mathrm{VI} \ldots \ldots \ldots \ldots \ldots \ldots \ldots \ldots \ldots . \ldots \ldots 2 \times \ldots \ldots \ldots$

$\mathrm{V}$ : Science \& Technology of Magnetic Oxides ............\$50 $\$ \ldots \ldots$

W: Chemical Aspects of Electronic Ceramics Processing .......\$52 $\times$ = $=\$$

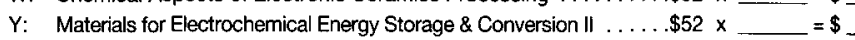

Z: Recent Advances in Catalytic Materials .............\$\$ $\$ 50 \times \ldots$

AA: Covalently Bonded Disordered Thin-Film Materials ........\$50 $\ldots$

DD: High-Pressure Materials Research $\ldots \ldots \ldots \ldots \ldots \ldots \ldots \ldots . \$ 52 \times \ldots \ldots \ldots$

EE: Electrically Based Microstructural Characterization II . . . . . .\$50 $\times$

FF: Surf.-Controlled Nanoscale Matls. for High-Added-Value Appls.. . . $\$ 50 \times \square=\$$

II: In Situ Process Diagnostics \& Intelligent Matls. Processing .......\$55 $\times$ = $\$$

$\mathrm{JJ}$ : Nondestructive Characterization of Matls. in Aging Systems ...\$48 $\times$

KK: Atomistic Mechanisms in Beam Synthesis \& Irrad. of Matls. . . .\$52 $\times-$

NN: Thin Films - Stresses and Mechanical Properties VII ........\$52 $\times$

Sub-Total

Sales Tax (PA residents $7 \%$ )

- Enter total here and in PAYMENT OPTIONS box. TOTAL \$

\section{- PAYMENT OPTIONS}

$\square$ Payment is enclosed. Make checks payable, in U.S. dollars, to Materials Research Society. Payment from outside the U.S. should be drawn on a correspondent U.S. bank

Credit card payment: $\square$ Visa $\square$ Master Card $\square$ Diners Club $\square$ American Express Card No. Exp.

Signature

\section{OPTIONAL TUTORIAL NOTES}

$\square$ FTG: Fundamentals of Solar Cells-Design and Material Issues ..................................\$25

FTI: Imaging with Room-Temperature Semiconductor Detectors .................................\$25

$\square$ FTU: Ferroelectric Thin Films ...................................................................................\$25

$\square$ FTW: Chemical Processing and Applications of Electronic

Ceramics-Chemical Vapor Deposition and Sol-Gel Processing ......................\$25

$\square$ FTf: Surface Control of Nanoscale Materials through

Clean Synthesis, Functionalization and Characterization.................................\$25

$\square$ FTj: Nondestructive Materials Evaluation-An Overview

Addressing Its Theory and Application......

Sub-Total

$\$$

Sales Tax (PA residents $7 \%$ ) \$

- Enter total here and in PAYMENT OPTIONS box. TOTAL \$

(Cost for notes will be $\$ 35$ after the preregistration deadline.)
A Meeting Preregistration (from left)
B Tutorial Notes (from left)
C Journal of Materials Research (from above)
D Proceedings (from above)

TOTAL FEES PAID\$

\section{CANCELLATION/REFUNDS}

970197

To cancel your registration, you must notify MRS in writing of your request for a refund. Refunds will be made upon receipt of this written notice, less a $\$ 25$ service charge. If you apply a portion of this refund to member dues or any MRS publications, the service charge will be waived. MRS will not honor requests made more than one calendar month after the close of the meeting. 